\title{
Platelet Rich Fibrin and Bone Graft in the Treatment of Intrabony Defect in Periodontitis Patients
}

\author{
Dr. Shilu Shrestha, ${ }^{1}$ Dr. Surendra Man Shrestha, ${ }^{1}$ Dr. Ameena Pradhan, ${ }^{1}$ Dr. Shreeya Aryal ${ }^{1}$ \\ ${ }^{1}$ Department of Periodontology and Oral Implantology, People's Dental College and Hospital, \\ Kathmandu, Nepal.
}

\begin{abstract}
Background: Porous hydroxyapatite and $\beta$-tricalcium phosphate ( $\beta$-TCP + HA) bone grafting material has resulted in clinically acceptable responses when used to fill the periodontal intrabony defects. PRF is an autologous leukocyte and platelet preparation that concentrates various polypeptide growth factors which therefore holds potential to be used as regenerative treatment for periodontal defects.

Aim: The purpose of this study was to evaluate clinical and radiographic outcomes in periodontal intrabony defects treated with platelet rich fibrin compared to alloplastic bone graft material.

Materials and Methods: Twenty subjects (10 subject per group, one site/subject) were treated either with platelet rich fibrin or alloplastic bone graft (30\% $\beta$-TCP+ 70\% HA). Primary clinical parameters: PD, CAL were taken at baseline, three months, six months and nine months post-operatively. Standardised radiographic data were collected at baseline, six months and nine months postoperatively.

Results: Preoperative parameters were similar for both groups. Postsurgical measurements revealed a greater reduction in pocket depth in bone graft group $(2.5 \mathrm{~mm})$, greater CAL gain $(2.2 \mathrm{~mm})$ and greater defect fill $(1.30 \mathrm{~mm})$ as compared to platelet rich fibrin group $(1.50 \mathrm{~mm}$, $1.6 \mathrm{~mm}$ and $0.80 \mathrm{~mm}$ respectively ) at nine months.

Conclusion: Treatment of intrabony defects with alloplast $(30 \% \beta$ TCP+ $70 \% \mathrm{HA})$ or platelet rich fibrin both resulted in a significant probing depth reduction, CAL gain and bone depth reduction, with significantly better improvement in bone graft group.

Keywords: Bone graft; clinical attachment loss; periodontitis; platelet rich fibrin; pocket depth; regeneration.
\end{abstract}

\section{INTRODUCTION}

Periodontal disease is characterised by the loss of connective tissue attachment with destruction of periodontal tissues. ${ }^{1}$ Periodontal regeneration is considered a multifactorial process $^{2,3}$ that occurs when the systemic and local conditions are favorable and when therapy is properly applied. ${ }^{4}$

Among variety of treatment modalities available, ${ }^{5}$ alloplastic bone substitutes like synthetic calcium phosphates: $\beta$-tricalcium phosphate ( $\beta$-TCP) and hydroxyapatite (HA) have their ability to bond bone minerals directly and to promote new bone formation by osteoconduction. ${ }^{6,7}$

\author{
Correspondence: \\ Dr. Shilu Shrestha \\ Department of Periodontology and Implantology, \\ People's Dental College and Hospital \\ email: shilu.sht@gmail.com \\ Citation
}

Shrestha S, Shrestha SM, Pradhan A, Aryal S. Platelet Rich Fibrin and Bone Graft in the Treatment of Intrabony Defect in Periodontitis Patients. J Nepal Soc Perio Oral Implantol. 2018;2(2):49-55.
Platelet-rich fibrin (PRF) described by Choukroun et $\mathrm{al}^{8}$ is a second-generation platelet concentrate that contains platelets and growth factors prepared from the patient's own blood free of any anticoagulant. ${ }^{1}$ PRF release polypeptide growth factors, such as transforming growth factor- $\beta 1$, platelet- derived growth factor, vascular endothelial growth factor and matrix glycoproteins (such as thrombospondin-1) and provides a three-dimensional fibrin matrix that may be utilized as a scaffold for a variety of procedures including the function as a barrier membrane., ${ }^{9,10}$

The purpose of this study was to report clinical and radiographic outcomes from treatment of periodontal intrabony defects with autologous PRF with open flap debridement compared to alloplastic bone graft material with open flap debridement.

\section{MATERIALS AND METHODS}

The study was conducted in the Department of Periodontology and Oral Implantology, People's Dental College and Hospital. The study design was approved by the Institutional Review Board of Institute of Medicine, Tribhuvan University, Kathmandu, Nepal. It was a randomised controlled clinical 


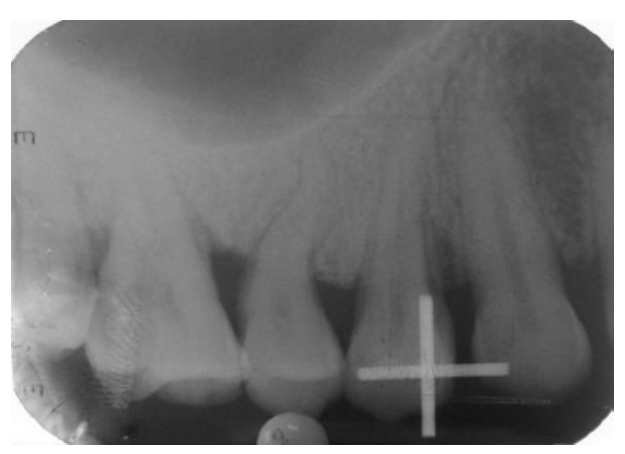

Figure 1a: Long cone parallel intraoral periapical view with radiographic stents.

trial. Twenty systemically healthy patients (12 women and 8 men, mean age 34.8 years) with interproximal, intrabony defects were enrolled in this study. Written informed consent was obtained from each patient and the patients were informed about the procedures to be performed and its benefits and risks were explained.

The inclusion criteria included patients with age 25-50 years with the presence of interproximal intrabony defects in maxillary and mandibular premolars and molars with probing depths 6-8 $\mathrm{mm}$, after the completion of phase I therapy. Osseous defects needed to have two or three walls. One-wall defects and interdental craters were excluded from the study. The plaque index achieved following initial therapy had to be $<1 .{ }^{11}$ Radiographic evidence of intrabony defects of 3-4 mm had to exist as revealed by periapical films taken with the long-cone parallel technique. ${ }^{12}$

The exclusion criteria were: the patients with systemic disease and under medications known to interfere with periodontal healing and regeneration, and contraindication for periodontal surgery, patients who were pregnant or lactating and patients undergoing orthodontic treatment, patients with smoking habit or tobacco chewing habit and surgical periodontal therapy in the preceding six months. Tooth with mobility of Grade II, III, endo-perio lesion and third molars were also excluded.

Initial therapy consisted of scaling and root planing using Gracey curettes and an ultrasonic device. Patient education,

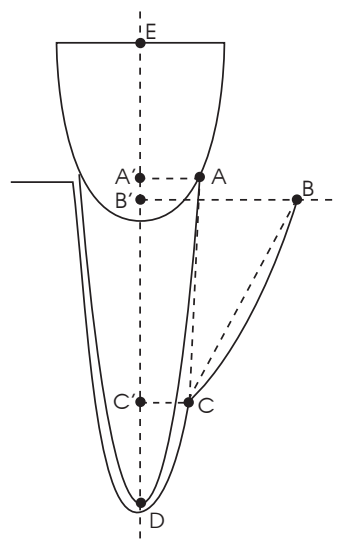

Figure 1b: Representation of reference points for radiographic analysis: A1-B1, horizontal (suprabony) component; B1-C1, vertical (intrabony) component of the defect.

motivation and detailed oral hygiene instructions were given. Patients were recalled after one week for review. Patients under the inclusion criteria were recalled. The defects were randomly assigned into two groups where group 1 were patients to be treated with autologous platelet rich fibrin and group 2 were patients to be treated with bone graft. Patients were not blinded for allocation to a particular group and treatment.

The patients were allocated according to the inclusion criteria. Pre-surgical clinical parameters measurements were done by University of North Carolina 15 (UNC-15) Hu-Friedy, Chicago, IL, USA periodontal probe. An individual occlusal stent was fabricated of cold cured acrylic resin on a cast model. Prior surgery, clinical parameters Plaque Index (PI), ${ }^{11}$ Oral Hygiene Index (OHI), Gingival Index (GI), Probing depth (PD), Clinical attachment loss (CAL) were measured.

Long cone paralleling technique with radiographic stent was used to obtain standardised radiographs. For the measurement of bone defect, criteria described by Schei et al 1959, ${ }^{13}$ and Bjorn et al 1969, ${ }^{14}$ were followed. (Figure 1a, 1b)

The PRF was produced according to the protocol developed by Choukroun et al $2001 .{ }^{8}$ Immediately before the surgical procedure, $10 \mathrm{ml}$ of blood was drawn from the subjects by venipuncture of the antecubital vein. The blood sample was collected in sterile glass test tubes $(10 \mathrm{ml})$ and were immediately centrifuged (ISO9001, ISO 13485, GMP,FDA, CE MARKING, Gemmy Industrial Corp. Taipei, Taiwan) at 3000
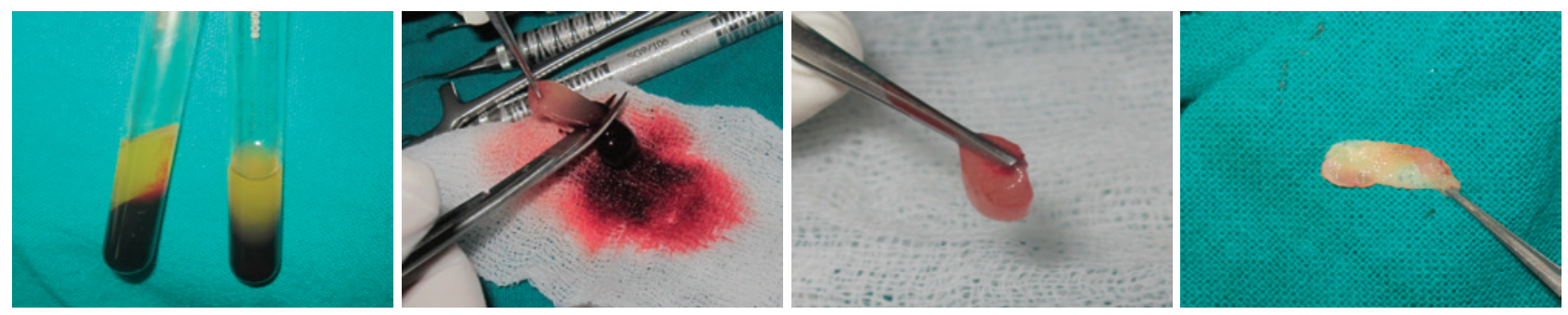

Figure 2: Platelet rich fibrin gel and membrane preparation. 

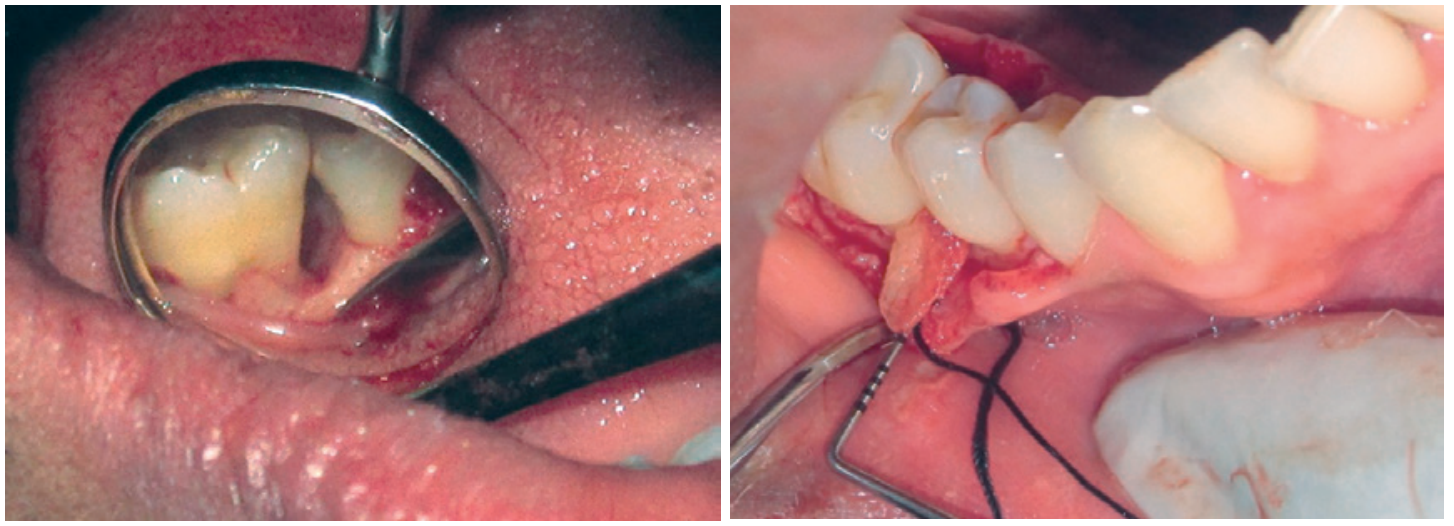

Figure 3: Platelet rich fibrin gel and membrane placed in the infrabony defect in lower right first molar after debridement.

rpm for 10 minutes. The centrifuged blood mass presented with a structured fibrin clot in the middle fraction of the tube, between the red blood cells (RBC) corpuscle layer on the lower fraction and the straw-colored acellular plasma on upper fraction. The fibrin clot could easily be removed from the tube and shaped freely, and was used immediately after its collection (Figure 2).

The upper portion of the test tube containing the acellular plasma was removed. The middle portion containing the fibrin clot was then removed and scraped off from the lower part containing the red blood cells base, preserving a small red blood cell layer, using sterile tweezers and scissors just after removal of platelet poor plasma (PPP). PRF was used immediately after its preparation.

A stable fibrin membrane was obtained by squeezing serum out of the PRF clot between two wet gauge pieces in order to take the form of a consistent autologous fibrin membrane, which will be applied over the treatment defects (Figure 2).

In Group 2, bone graft (GENOSS OSTEON II) of particle size $0.2-0.5 \mathrm{~mm}$, volume 0.25 cc was mixed with as per the manufacturer's instructions.

One surgeon performed all surgeries. Each patient contributed only one intrabony defect. Patients were made to rinse with $0.2 \%$ chlorhexidine digluconate and povidine iodine solution was used to carry out extraoral antisepsis. The surgical area was anaesthetised with $2 \%$ lidocaine. Buccal and lingual intracrevicular incisions were made extending to the 1-1 adjacent tooth mesially and distally. Full thickness mucoperiosteal flaps were reflected. Extreme care was exercised to preserve as much marginal and interproximal soft tissue as possible to achieve better closure of the grafted sites.

Meticulous defect debridement and root planing was carried out carefully. To avoid the displacement of PRF, a 4-0 non-absorbable black silk surgical suture was passed through buccal and lingual flap before placement of the mixture. Prepared PRF was taken. Size reduction was done by cutting the PRF clot into appropriately sized pieces to fit the bony defect to the coronal margin of the alveolar crest. A membrane of compressed PRF was trimmed and adapted over the grafted defect (Figure 3). Membrane was extended over the periphery of the defect in the buccal and lingual directions. The mucoperiosteal flaps were repositioned and secured in place using the suture. The interrupted suture was placed. Periodontal dressing was not utilised.

Surgical procedures for Group 2 were performed with the same surgical protocol. Bone graft of particle size 0.2-0.5 mm,
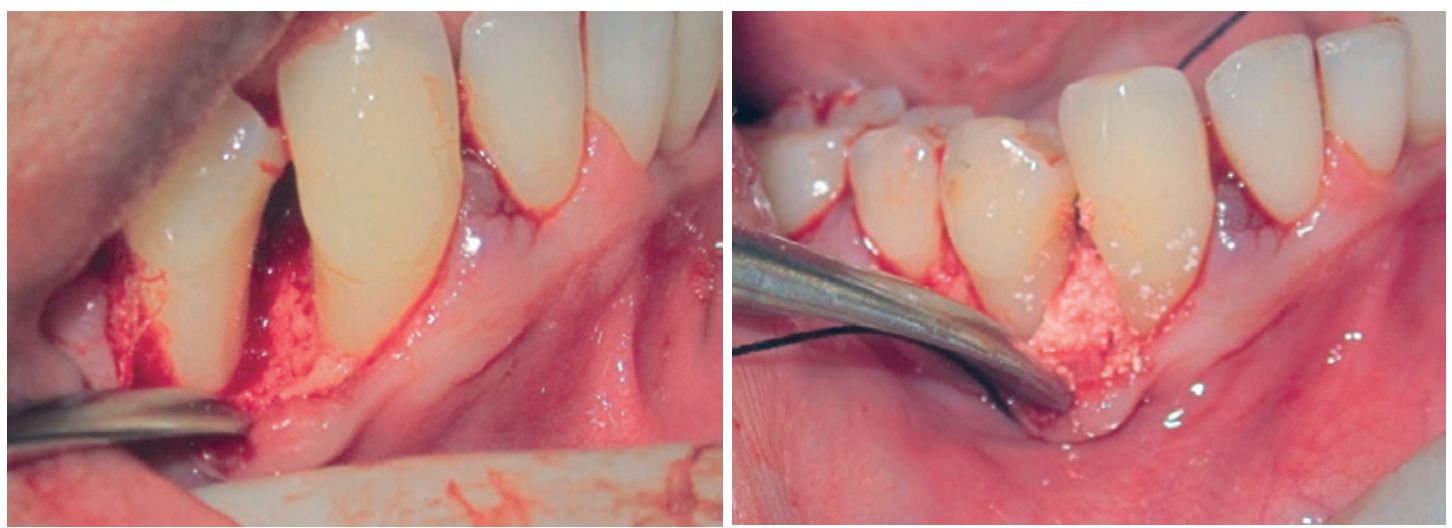

Figure 4: Placement of bone graft in infrabony defect in lower right first premolar after debridement. 


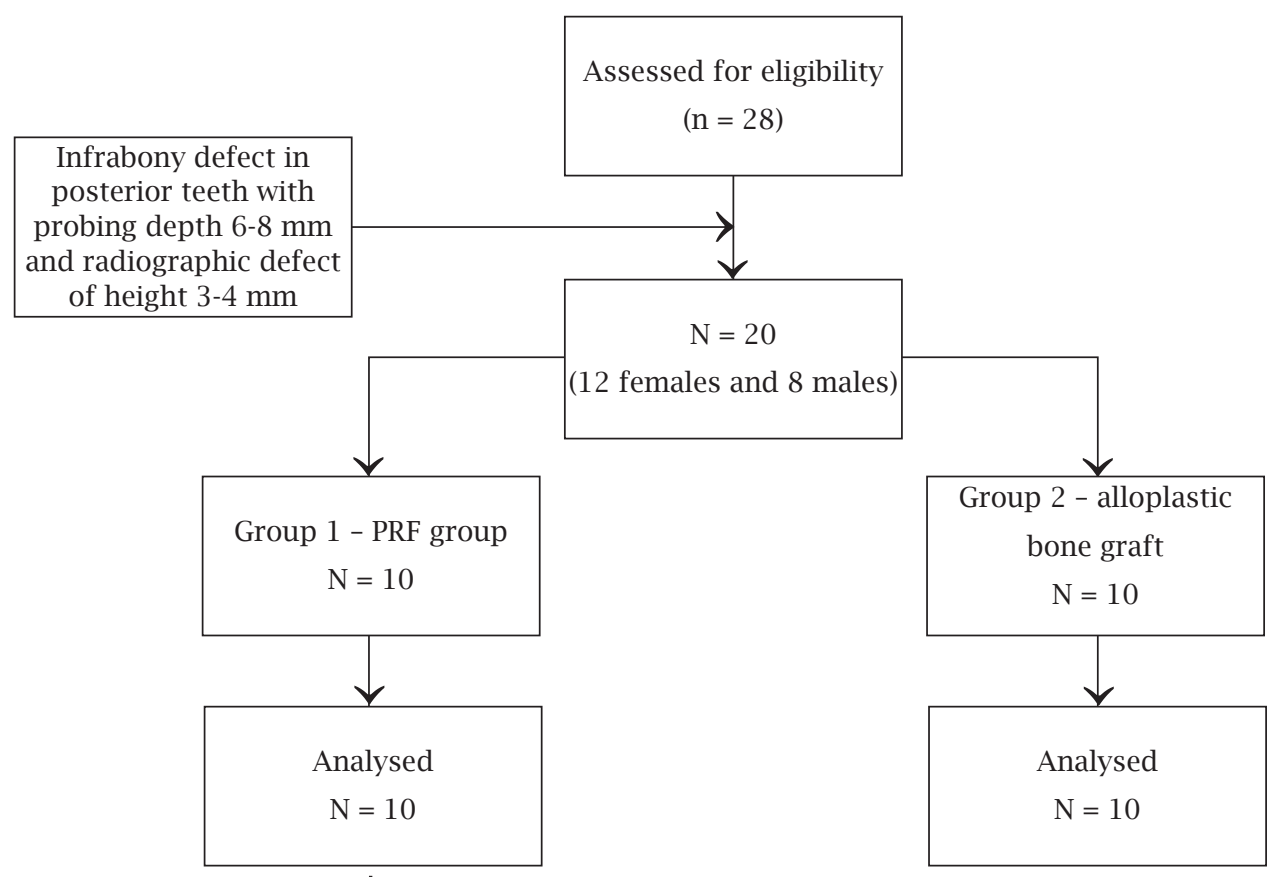

Figure 5: Study flow chart.

volume 0.25 cc was mixed with sterile saline as per the manufacturer's instructions (Figure 4). Suitable antibiotics and analgesics were prescribed.

Sutures were removed two weeks postoperatively. All clinical measurements were repeated at one, three, six and nine months after the initial surgery. Clinical parameters (probing depth and clinical attachment level) measurements were repeated with previously used acrylic stents. For hard tissue re-evaluation, intraoral periapical radiograph of the same study site was carried out and bone defect measurement was reassessed at six and nine months.

Power analysis determined that a sample size of 10 defects per protocol group was sufficient to detect a clinically significant mean difference at the 0.05 level with power of $80 \%$, taking, $10 \%$ amplification for non-response and $20 \%$ for loss to Follow-up. Clinical measurements for each group were averaged (means \pm standard deviation). The comparison of clinical outcome and radiological outcome between the groups at different time intervals were done with the help of Independent t-test and Paired t-test after checking for normal distribution of the data.

\section{RESULTS}

All 20 patients completed the study and healing was uneventful in both the groups. There were no significant differences in pocket depth between the two groups at baseline. Both PRF and bone graft groups showed significant pocket depth reduction and clinical attachment level gain at nine month compared with baseline. Mean pocket depth in PRF group at baseline was $7.20 \pm 0.78$ which was reduced to $5.70 \pm 1.16$ at nine months. Similarly mean pocket depth in bone graft group was $6.60 \pm 0.69$ at baseline that was reduced to $4.10 \pm 0.99$ at nine months which was statistically significant. Changes in pocket depth are reported in Table 1 .

Changes in clinical attachment level are reported in Table 2. PRF group had mean clinical attachment loss of $7.30 \pm 0.82$ at baseline which was reduced to $5.70 \pm 1.41$ in nine months. Bone graft group had mean clinical attachment loss of 6.70

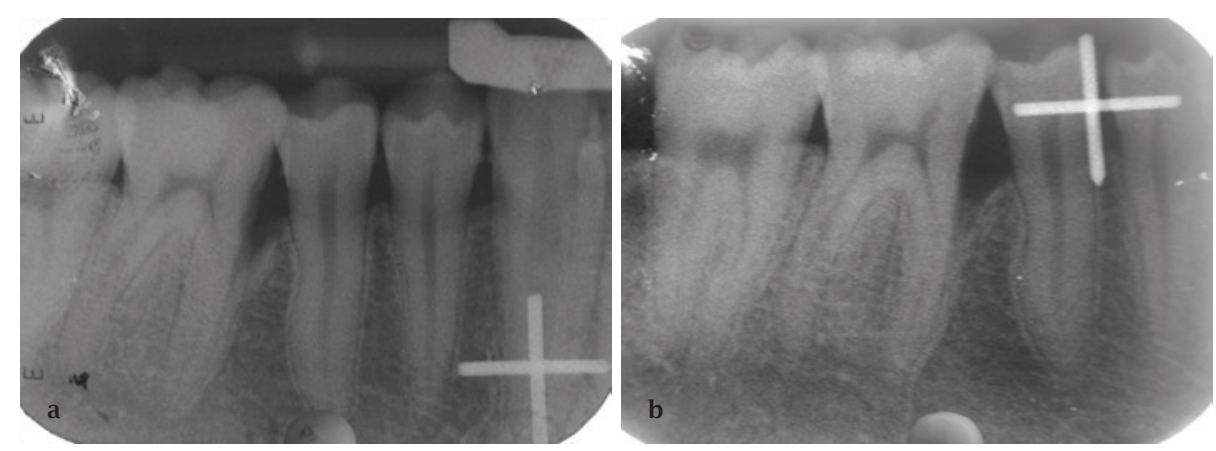

Figure 6a and 6b: Long cone parallel IOPA of a patient treated with PRF at baseline and nine months in lower right first molar. 


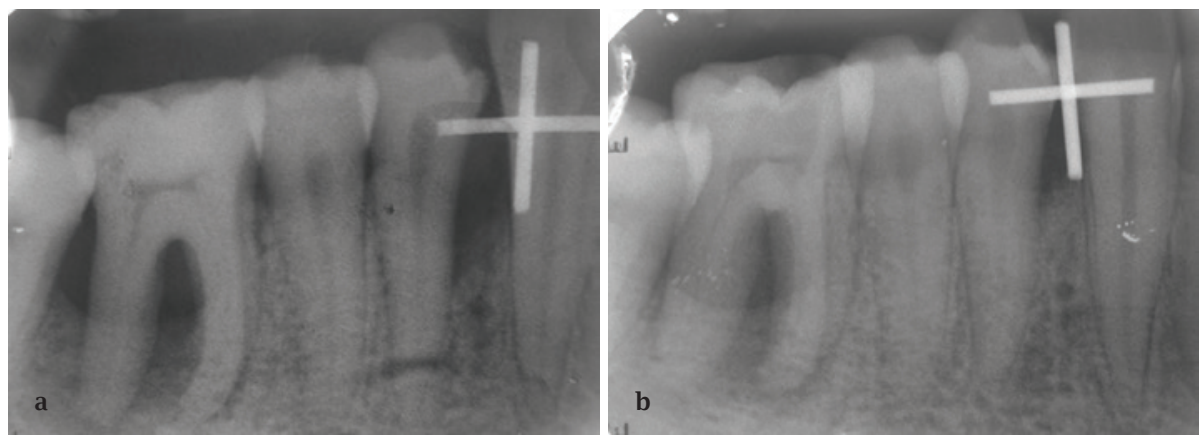

Figure 7a and 7b: Long cone parallel IOPA of a patient treated with bone graft at baseline and nine months in lower right first premolar.

Table 1: Comparison of pocket depth reduction in two study groups.

\begin{tabular}{|l|c|c|c|c|c|}
\hline \multicolumn{1}{|c|}{ Pocket depth $(\mathrm{mm}$, mean \pm SD) } & Baseline & 3 months & 6 months & 9 months & P value \\
\hline PRF & $7.20 \pm 0.78$ & $6.40 \pm 1.43$ & $6.10 \pm 1.44$ & $5.70 \pm 1.16$ & $0.002^{*}$ \\
\hline Bone graft & $6.60 \pm 0.69$ & $4.90 \pm 1.19$ & $4.20 \pm 1.39$ & $4.10 \pm 0.99$ & $<0.001^{*}$ \\
\hline P value & 0.089 & $0.020^{*}$ & $0.008^{*}$ & $0.004^{*}$ & \\
\hline
\end{tabular}

"statistically significant.

Table 2: Comparison of clinical attachment level in two study groups.

\begin{tabular}{|l|c|c|c|c|c|}
\hline Clincal Attachment Loss $(\mathrm{mm}$, mean \pm SD) & Baseline & 3 months & 6 months & 9 months & P value \\
\hline PRF & $7.30 \pm 0.82$ & $6.40 \pm 1.43$ & $6.10 \pm 1.44$ & $5.70 \pm 1.41$ & $0.007^{*}$ \\
\hline Bone graft & $6.70 \pm 0.82$ & $5.00 \pm 1.15$ & $4.20 \pm 1.39$ & $4.50 \pm 1.43$ & $0.001^{*}$ \\
\hline P value & 0.121 & $0.027^{*}$ & $0.008^{*}$ & 0.076 & \\
\hline
\end{tabular}

*statistically significant.

Table 3: Comparison of radiographic defect depth in two study groups.

\begin{tabular}{|l|c|c|c|c|}
\hline \multicolumn{1}{|c|}{ Bone Defect $(\mathrm{mm}$, mean $\pm \mathrm{SD})$} & Baseline & 6 months & 9 months & P value \\
\hline PRF & $3.50 \pm 0.52$ & $2.8 \pm 0.48$ & $2.7 \pm 0.42$ & $<0.006^{*}$ \\
\hline Bone graft & $3.40 \pm 0.45$ & $2.30 \pm 0.34$ & $2.10 \pm 0.45$ & $<0.001^{*}$ \\
\hline P value & 0.656 & $0.016^{*}$ & $0.007^{*}$ & \\
\hline
\end{tabular}

*statistically significant.

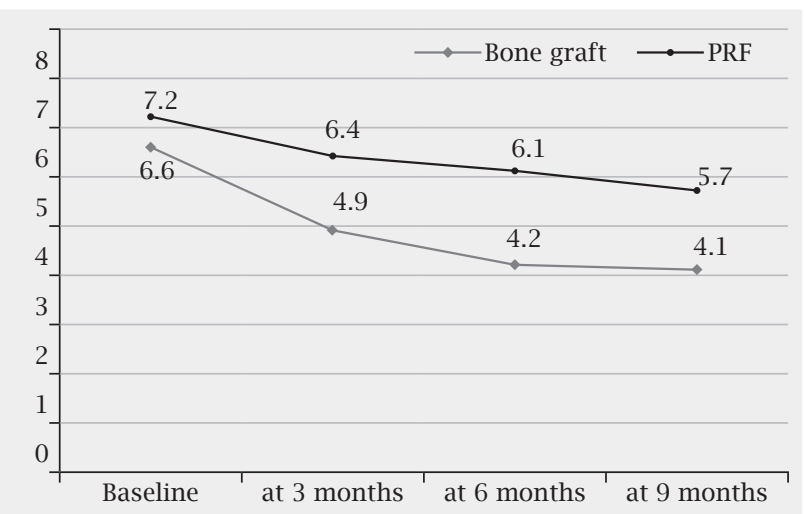

Figure 8: Time trend of change in mean values of probing depth among the two study groups ( $\mathrm{mm})$.

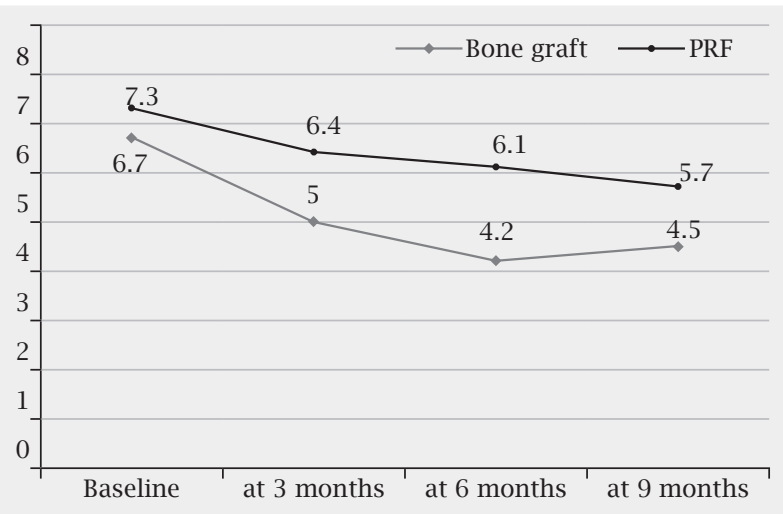

Figure 9: Time trend of mean values of CAL among two study groups (mm).

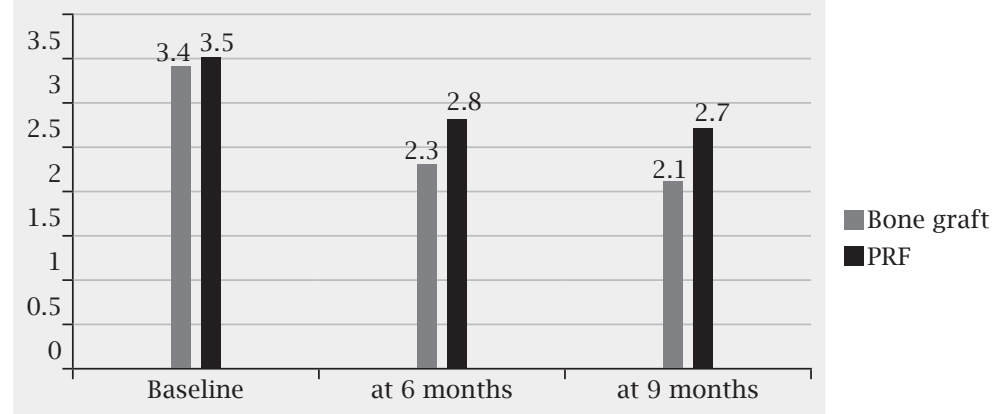

Figure 10: Bone defect levels among the two study groups (mm). 
\pm 0.82 at baseline which was reduced to $4.50 \pm 1.43$ in nine months. While, the clinical attachment gain were different in favour of bone graft group than platelet rich fibrin group (Table 1, 2). Figure 8 and Figure 9 shows the diagrammatic difference of the comparison between two groups.

Table 3 reports the changes in defect fill between the two groups where bone graft group shows significant reduction in radiographic defect depth $(3.40 \pm 0.45$ at baseline: $2.10 \pm 0.45$ in nine months; P value $<0,001$ ) as compared to PRF group (3.50 \pm 0.52 at baseline: $2.7 \pm 0.42$ in nine months; $\mathrm{P}$ value $<0.006)$. Diagrammatic illustration is shown in Figure 10.

\section{DISCUSSION}

This randomised clinical trial was designed to compare the treatment of periodontal vertical defects with platelet-rich fibrin and conventional open flap debridement with an active treatment of alloplastic bone graft material $(30 \% \beta$ Tricalcium Phosphate $+70 \%$ Hydroxyapatite) and open flap debridement. In total, 20 subjects (10 subjects per group, one site per subject) were treated. The treatment protocol emphasised the principles of careful soft tissue handling, wound stability and infection control. No uneventful healing and postoperative complication was observed in any of the studied groups. The uneventful healing in patients was in agreement with various previous studies ${ }^{15-17}$ thus supporting the excellent biocompatibility and enhanced wound healing properties of PRF and alloplast bone graft.

In the present study, defects treated in both groups showed significant improvements in all clinical parameters compared between baseline and nine months. However, there was more PD reduction $(2.5 \mathrm{~mm})$ and CAL gain $(2.2$ $\mathrm{mm})$ in the alloplast $(\beta \mathrm{TCP}+\mathrm{HA})$ treated group compared with the subjects treated with platelet rich fibrin (PD reduction $1.6 \mathrm{~mm}$; CAL gain $1.6 \mathrm{~mm}$ ). The present study also reflects the intrabony defect fill in the bone graft group $(1.3 \mathrm{~mm})$ is higher than the subjects that were treated with PRF (0.8 mm).

To our knowledge, there are very less studies reporting the comparison of autologous PRF and alloplastic bone graft in the treatment of vertical intrabony defects. Therefore, a direct comparison with other studies is not possible.

The result of our study supports the study conducted by Chadwick et al in $2016^{18}$ who reported changes in clinical attachment level and bone fill of periodontal intrabony defects treated with demineralized freeze dried bone allograft (DFDBA) compared to platelet-rich fibrin (PRF) in humans. He concluded that both treatment groups had significant gains in CAL as well as bone fill, with no significant differences in outcomes between groups but there was slight better outcomes in DFDBA group than the PRF group.

Thorat et $\mathrm{al}^{16}$ investigated the clinical and radiological effectiveness of autologous PRF in the treatment of intrabony defects of chronic periodontitis patients. He reported a greater reduction in pocket depth, more gain in clinical attachment level and greater intrabony defect fill at sites treated with PRF than those treated with open flap debridement alone and the difference was found to be statistically significant.

Despite the fact that PRF is a denser and firmer agent than other biological preparations, it is still considered non-rigid to a degree that its space-maintaining ability in periodontal defects is not ideal and it may be like regenerative adjuncts. ${ }^{18}$ Subjectively, the opinion of the clinicians in various studies was that PRF was more difficult to handle than bone grafts. The slippery consistency of PRF made it difficult to keep the material within the defect after placement. The lack of rigidity and space making capacity of the PRF material may make it more difficult to contain in the defect. ${ }^{16}$

Lekovic in $2012^{5}$ examined the suitability of autologous PRF as regenerative treatment for periodontal intrabony defects in humans and to examine the ability of bovine porous bone mineral (BPBM) to augment the regenerative effects exerted by PRF. Postsurgical measurements revealed a significantly greater reduction in pocket depth, greater attachment gain and greater defect fill in the PRF-BPBM group than in the PRF group. He concluded that PRF can improve clinical parameters associated with human intrabony periodontal defects, and BPBM has the ability to augment the effects of PRF in reducing pocket depth, improving clinical attachment levels and promoting defect fill.

In our study, PRF group showed statistically significant pocket depth reduction and clinical attachment level gain only at nine months while the defect fill was statistically significant at both six and nine months. Different subjects may have distinct healing responses and/or the employed surgical technique may jeopardize the regenerative potential. ${ }^{19}$ This gain might have been the result of true periodontal regeneration via new attachment or, alternatively, healing by repair, which implies the presence of a long junctional epithelium between the newly regenerated tissues and the root surface.

Defect morphology plays a major role in healing following periodontal-regenerative treatment of intrabony defects. ${ }^{19-22}$ In the present study, the defect depth was measured, but the width and angle of the vertical defect weren't measured. Wider defects have been associated with reduced amounts of clinical attachment level and bone gain at one year. ${ }^{19,21,23}$

Radiographic evaluation is a noninvasive examination for bony defects repair. However, bone fill data derived from surgical re-entry are important to substantiate routine postoperative measurement data. In addition, histology of the treated periodontal intrabony defects is the only reliable method to determine the nature of the periodontal soft and hard tissue interface. ${ }^{24}$ 
Although results of the present study demonstrates that the use of autologous PRF and alloplast were effective in the treatment of two or three wall intrabony defect with an uneventful healing of the sites, our results are based on single-centered study with a small sample size. Future research is needed, including histologic evaluation of defects treated with PRF and alloplast to determine if periodontal regeneration is obtained. Studies with larger sample sizes, multi-centered and longer follow-ups would also be valuable to help guide treatment decisions.

\section{CONCLUSION}

The result of this study indicates that both treatment resulted in reduction in pocket depth, gain in clinical attachment level and defect fill compared with baseline, but there was greater reduction in pocket depth, more clinical attachment level gain and greater vertical defect fill at sites treated with alloplastic bone graft with open flap debridement than the sites treated with PRF with open flap debridement.

\section{ACKNOWLEDGEMENTS}

The authors would like to thank Department of Periodontology and Implantology of People's Dental College and Hospital for the support.

\section{REFERENCES}

1. Preeja C, Arun S. Platelet-rich fibrin: Its role in periodontal regeneration. Saudi J Dent Res. 2014;5(2):117-22.

2. William VG. The potential role of growth and differentiation factors in periodontal regeneration. J. Periodontol. 1996;67:545-53.

3. Melcher AH. On the repair potential of periodontal tissues. J Periodontol. 1976;47(5):256-60.

4. Cortellini P, Tonetti MS. Clinical concepts for regenerative therapy in intrabony defects. Periodontol 2000. 2015;68(1):282-307.

5. Lekovic V, Milinkovic I, Aleksic Z, Jankovic S, Stankovic P, Kenney EB, Camargo PM. Platelet-rich fibrin and bovine porous bone mineral vs. platelet-rich fibrin in the treatment of intrabony periodontal defects. J Periodont Res. 2012;47(4):409-17.

6. Low SB, King CJ, Krieger J. An evaluation of bioactive ceramic in the treatment of periodontal osseous defects. Int J Periodontics Restorative Dent. 1997;17(4):358-67.

7. Giannoudis PV, Dinopoulos H, Tsiridis E. Bone substitutes: an update. Injury. 36 Suppl 3:S20-7.

8. Choukroun J, Adda F, Schoeffler C, Vervelle AP. Une opportunité en paro-implantologie: le PRF. Implantodontie. 2001;42:55-62.

9. Fujioka-Kobayashi M, Miron RJ, Hernandez M, Kandalam U, Zhang Y, Choukroun J. Optimized platelet-rich fibrin with the low-speed concept: growth factor release, biocompatibility, and cellular response. J Periodontol. 2017;88(1):112-21.

10. Dohan Ehrenfest DM, de Peppo GM, Doglioli P, Sammartino G. Slow release of growth factors and thrombospondin-1 in Choukroun's platelet-rich fibrin (PRF): a gold standard to achieve for all surgical platelet concentrates technologies. Growth Factors. 2009;27(1):63-9

11. Silness J, Löe H. Periodontal disease in pregnancy II. Correlation between oral hygiene and periodontal condition. Acta Odont Scand. 1964;22(1):121-35.

12. Updegrave WJ. The paralleling extension-cone technique in intraoral dental radiography. Oral Surg Oral Med Oral Pathol. 1951;4(10):1250-61.

13. Schei O, Waerhaug J, Lovdal A, Arno A. Alveolar bone loss as related to oral hygiene and age. J Periodontol. 1959;30(1):7-16.

14. Björn HI, Halling AR, Thyberg HÅ. Radiographic assessment of marginal bone loss. Odontologisk Revy. 1969;20(2):165-79

15. Sharma A, Pradeep AR. Treatment of 3-wall intrabony defects in patients with chronic periodontitis with autologous platelet-rich fibrin: a randomized controlled clinical trial. J Periodontol. 2011;82(12):1705-12.

16. Thorat M, Pradeep AR, Pallavi B. Clinical effect of autologous platelet-rich fibrin in the treatment of intra-bony defects: a controlled clinical trial. J Clin Periodontol. 2011;38(10):925-32.

17. Czuryszkiewicz-Cyrana J, Banach J. Autogenous bone and platelet-rich plasma (PRP) in the treatment of intrabony defects. Adv Med Sci. 2006;51(Suppl 1):26-30.

18. Chadwick JK, Mills MP, Mealey BL. Clinical and radiographic evaluation of demineralized freeze-dried bone allograft versus platelet-rich fibrin for the treatment of periodontal intrabony defects in humans. J Periodontol. 2016;87(11):1253-60.

19. Tonetti MS, Pini-Prato G, Cortellini P. Periodontal regeneration of human intrabony defects. IV. Determinants of healing response. J Periodontol. 1993;64(10):934-40

20. Ehmke B, Rüdiger SG, Hommens A, Karch H, Flemmig TF. Guided tissue regeneration using a polylactic acid barrier. J Clin Periodontol. 2003;30(4):368-74.

21. Garrett S, Loos B, Chamberlain D, Egelberg J. Treatment of intraosseous periodontal defects with a combined adjunctive therapy of citric acid conditioning, bone grafting, and placement of collagenous membranes. J Clin Periodontol. 1988;15(6):383-9.

22. Silvestri M, Sartori S, Rasperini G, Ricci G, Rota C, Cattaneo V. Comparison of infrabony defects treated with enamel matrix derivative versus guided tissue regeneration with a nonresorbable membrane. J Clin Periodontol. 2003;30(5):386-93.

23. Cortellini P. Radiographic defect angle influences the outcomes of GTR therapy in intrabony defects. J Dent Res. 1999;78:2208.

24. Nasr HF, Aichelmann-Reidy ME, Yukna RA. Bone and bone substitutes. Periodontol 2000. 1999;19(1):74-86 